\title{
GRAM STAIN DETECTION OF INFECTION DURING REVISION ARTHROPLASTY
}

\author{
GEORGE F. CHIMENTO, SIMON FINGER, ROBERT L. BARRACK
}

From Tulane University, New Orleans, USA

$\mathbf{W}$

e reviewed 194 revision arthroplasties of the hip and knee performed over a ten-year period. The results of intraoperative Gram staining were available in $169(87 \%)$. Thirty-two were found to be infected (11 hips and 21 knees) and 137 had no evidence of infection.

Intraoperative Gram staining was negative in all 169 cases. The method therefore had a sensitivity of $0 \%$ for detecting infection. We conclude that the absence of organisms on intraoperative Gram staining during revision arthroplasty does not confirm the absence of infection.

J Bone Joint Surg [Br] 1996;78-B:838-9.

Received 21 December 1995; Accepted after revision 21 March 1996

Infection after revision arthroplasty is a disastrous complication. Adequate preoperative investigations must be done before the operation to ensure that there is no infection. They should include laboratory studies, plain radiography and occasionally scintigraphy and joint aspiration (Harris and Barrack 1993). In patients in whom the presence of infection is suspected clinically but whose preoperative assessment has been inconclusive, intraoperative tests such as Gram staining may be used to determine the presence or absence of infection. Our aim was to correlate the results of Gram staining performed on swabs taken during revision hip and knee arthroplasty on patients with known clinical infection to determine the accuracy of the prediction of infection.

\section{PATIENTS AND METHODS}

We reviewed all patients who had had revision hip or knee arthroplasties performed at Tulane University Medical Centre between 1982 and 1992. The 194 patients included

G. F. Chimento, MD, Senior Resident in Orthopaedic Surgery

S. Finger, MD, Resident in Orthopaedic Surgery

R. L. Barrack, MD, Professor of Orthopaedic Surgery and Director, Adult Reconstructive Surgery

Tulane University School of Medicine, 1430 Tulane Avenue, SL32, New Orleans, Louisiana 70112, USA.

Correspondence should be sent to Dr R. L. Barrack.

(C)1996 British Editorial Society of Bone and Joint Surgery 0301-620X/96/51252\$2.00 those who had had reimplantation of another component and others who had an excision arthroplasty for known or suspected infection. Complete clinical data, including the results of intraoperative Gram staining, were available for 169 (87\%); there were 101 hips and 68 knees. In the remaining 25 patients, complete records were not available or Gram staining had not been performed. None of these patients had had preoperative clinical evidence of infection.

We reviewed the clinic and inpatient charts and operating notes to determine if the patient was infected at the time of revision and/or taking antibiotics. We also compared the intraoperative Gram stain and the final results of culture which had been obtained at operation. A patient was judged to be clinically infected at the time of revision surgery if the clinical course and intraoperative appearance were consistent with infection, which was confirmed by intraoperative cultures and/or histological examination according to previously published guidelines (Barrack and Harris 1993). Patients who had a single positive culture and no other evidence of infection based on the clinical course or tissue histology were considered to have a false-positive result. All patients judged to be infected had at least one positive intraoperative culture on solid media and/or histological confirmation of the presence of acute inflammation.

At our institution Gram staining is interpreted by a technician and the results are relayed to the theatre. It is negative if no organisms are seen. If polymorphonuclear leucocytes (PMNs) are present, they are grouped according to the number of PMNs per high-powered oil-immersion field (Table I).

\section{RESULTS}

Thirty-two of the cases were diagnosed as infected at the time of the revision operation and 137 had no clinical or histological evidence of infection. Of the 32 infected joints, 11 were hips and 21 were knees.

No bacteria were seen on the Gram stain in any of the revision procedures, including the 32 with documented infection.

The number of PMNs visualised per Gram stain were classified semiquantitatively as absent (0 PMNs), rare (1 to 5 PMNs in some, but not all high-powered fields), few (1 to 5 PMNs in all fields), moderate (5 to 10 PMNs in all fields), or numerous ( $>10$ in all fields). The number and percentage of 
Table I. Classification of polymorphonuclear leucocytes (PMNs) per oil-immersion field

\begin{tabular}{ll}
\hline Classification & Number of PMNs \\
\hline None & 0 \\
Rare & 1 to 5 in some fields \\
Few & 1 to 5 in all fields \\
Moderate & 5 to 10 in all fields \\
Numerous & $>10$ in all fields \\
\hline
\end{tabular}

culture, Gram staining and histological examination. Eftekhar (1993) stated that although the results of intraoperative Gram staining correlate poorly with the development of infection, an increased number of PMNs is evidence of infection. Our findings support this. A positive Gram stain for bacteria is a definite indication of infection, but in our series there were no such positive Gram stains in 32 infected cases. This confirms the findings of Athanasou et al (1995) who reported only four positive Gram stains in 23

Table II. Number and percentage of polymorphonuclear leucocytes present in the Gram stain in 169 cases

\begin{tabular}{lllccrr}
\hline & $\begin{array}{l}\text { None } \\
(\mathbf{0})\end{array}$ & $\begin{array}{l}\text { Rare } \\
(\mathbf{1} \text { to } \mathbf{5})\end{array}$ & $\begin{array}{l}\text { Few } \\
(\mathbf{1} \text { to 5) }\end{array}$ & $\begin{array}{l}\text { Moderate } \\
(\mathbf{5} \text { to 10) }\end{array}$ & $\begin{array}{l}\text { Numerous } \\
(>\mathbf{1 0})\end{array}$ & $\begin{array}{l}\text { Total } \\
\text { number }\end{array}$ \\
\hline Infected & 928.1 & 515.6 & 721.9 & 515.6 & 618.8 & 32 \\
Not infected & 6346.0 & 3827.7 & 1913.9 & 118.0 & 64.4 & 137 \\
Total & 7242.6 & 4325.4 & 2615.4 & 169.5 & 127.1 & 169 \\
\hline
\end{tabular}

each seen in infected and non-infected cases are shown in Table II. We determined the significance of the number of PMNs present using the chi-squared test. Gram stains in which no PMNs were seen were associated with an absence of infection $(p<0.046)$. Those classified as rare were suggestive of the absence of infection, but this was not significant $(\mathrm{p}>0.083)$ and those classified as few or moderate had no clinical correlation and no statistical significance. Gram stains classified as showing numerous PMNs were significantly more common in infected than non-infected cases $(\mathrm{p}<0.05)$. The definition of more than ten PMNs per high-powered field (numerous) as positive for infection, gives a sensitivity of $18.8 \%$, a specificity of $95.6 \%$, an accuracy of $81 \%$, a positive predictive value of $50 \%$ and a negative predictive value of $83 \%$. Although numerous PMNs were seen more often in infected cases, this finding accurately predicted infection in only half these cases.

\section{DISCUSSION}

The Gram stain was devised in 1884 by Dr Christian Gram, and has undergone countless modifications (Kopeloff and Beerman 1922). The properties of the bacterial cell wall determine whether the organism will be Gram-positive or Gram-negative and have a role in the choice of antibiotic when infection occurs. Gram staining has been advocated as a quick method of determining the presence or absence of bacteria by histological examination. A specimen must have a bacterial density of at least ten organisms per millilitre, however, to visualise an organism under the oilimmersion lens of a microscope (Eftekhar 1993).

During revision surgery, we send samples routinely for cases of infection. A negative intraoperative Gram stain is therefore a poor predictor of the absence of infection.

The association between the presence of acute inflammatory cells and infection was established by Mirra et al (1976), who described the presence of five or more PMNs per field in swabs of infected arthroplasties and the absence of PMNs in non-infected revisions. Their findings were based on permanent histological sections, but in our study the presence and number of PMNs on Gram staining did not correlate strongly with the presence or absence of infection.

Our results show that the absence of organisms on a Gram stain is not a reliable indication of the absence of infection. Any decision to proceed with revision of an arthroplasty should not be based on the findings of an intraoperative Gram stain.

No benefits in any form have been received or will be received from a commercial party related directly or indirectly to the subject of this article.

\section{REFERENCES}

Athanasou NA, Pandey R, De Steiger R, Crook D, McLardy Smith P. Diagnosis of infection by frozen section during revision arthroplasty. J Bone Joint Surg [Br] 1995;77-B:28-33.

Barrack RL, Harris WH. The value of aspiration of the hip joint before total hip arthroplasty. J Bone Joint Surg [Am] 1993;75-A:66-76.

Eftekhar NS. Postoperative wound infection. In: Total hip arthroplasty. St Louis, etc: Mosby, 1993:1457-504.

Gram C. Ueber die isolirte Färbung der Schizomyceten in Schnitt- und Trockenpräparaten. Fortschr Med 1884;2:185-9.

Harris WH, Barrack RL. Contemporary algorithms for evaluation of the painful total hip replacement. Orthop Rev 1993;22:531-9.

Kopeloff N, Beerman P. Modified Gram stains. J Infect Dis 1922;31: 480-2.

Mirra JM, Amstutz HC, Matros M, Gold R. The pathology of the joint tissues and its clinical relevance in prosthesis failure. Clin Orthop 1976;117:221-40. 